\title{
Differential Form of the Skornyakov-Ter-Martirosyan Equations
}

\author{
F. M. Pen'kov \\ Joint Institute for Nuclear Research, Dubna, Russia and \\ Institute of Nuclear Physics, Almaty, Kazakhstan \\ W. Sandha: \\ Physikalisches Institut, Universität Bonn, Bonn, Germany
}

(Dated: November 2, 2018)

\begin{abstract}
The Skornyakov-Ter-Martirosyan three-boson integral equations in momentum space are transformed into differential equations. This allows us to take into account quite directly the Danilov condition providing selfadjointness of the underlying three-body Hamiltonian with zero-range pair interactions. For the helium trimer the numerical solutions of the resulting differential equations are compared with those of the Faddeev-type AGS equations.

PACS numbers: 03.65.Nk, 11.10.Jj, 21.45.+v
\end{abstract}

\section{INTRODUCTION}

The discovery of a weekly bound state of two helium atoms [1] and problems concerning the stability of Bose condensates of alkali atoms (see, e.g., [2]) stimulated many calculations of the properties of three-particle systems determined by pair interactions with a large scattering length $a_{0}$ compared to the range $r_{0}$ of the pair forces,

$$
\frac{r_{0}}{a_{0}} \ll 1 .
$$

On the one hand, this condition poses problems in the numerical solution of the Faddeev equations with realistic atomatom potentials, on the other hand it forms the basis of the zero-range model of the two-body interaction $\left(\left(r_{0} \rightarrow 0\right)\right)$. This model determines the motion of particles beyond the pairinteraction region, admits an analytic simplification, and can be used for the description of real physical systems provided the condition (1) is fulfilled.

The zero-range model (ZRM) for three-body systems, though having obvious advantages and wide application, has a considerable drawback. The Hamiltonian of these systems is not self-adjoint (see, for example, [3] ) and the Schrödinger equation has quadratically integrable solutions at any energy. This fact was pointed out in [4] in analyzing poor attempts to solve the nd-scattering problem with the use of the Skornyakov-Ter-Martirosyan (STM) integral equations [5] within the zero-range model of nucleon-nucleon interaction. In particular, at large momenta $k$ of the relative motion of a particle and a pair the asymptotic behavior of the wave function was shown to have at any energy $Z$ the form

$$
\varphi(k, Z)=A \frac{\sin \left(\mu_{0} \ln (k)\right)}{k^{2}}+B \frac{\cos \left(\mu_{0} \ln (k)\right)}{k^{2}}+o\left(\frac{1}{k^{2}}\right) .
$$

Here, the constant $\mu_{0}$ depends on the ratio of the particle masses, and only the coefficients $A$ and $B$ depend on the energy. To determine the three-body spectrum, Danilov [4] suggested to use the relation between the coefficients $A(Z)$ and $B(Z)$ with an energy-independent parameter $\gamma$,

$$
A(Z)=\gamma B(Z)
$$

following from the orthogonality condition of eigenfunctions. In the same year Minlos and Faddeev [3] showed that the Danilov condition is a special case among possible extensions of the Hamiltonian to a self-adjoint one. Even after the extension, a solution to the STM equation has one free parameter. This opens the possibility of describing real three-body systems by adjusting the free parameter to a known spectral point [4].

Unfortunately, the Danilov condition for an unambiguous solution of the STM equations practically cannot be used in numerical calculations. But it was used for an analytic investigation of the ZRM three-body spectrum [6]. In this reference it has been shown that a three-body collapse (the Thomas effect [7]) is a specific feature of the ZRM.

There are several approaches using the ZRM beyond the scope of the STM equations, among them the adiabatic expansion in configuration space (see, e.g., [8]). Just in this approach the so-called Efimov effect was observed, i.e., the fact that three-body spectra concentrate on zero total energy if the two-body scattering length $\left|a_{0}\right|$ tends to infinity [9]. The result of adiabatic expansions is an infinite system of differential equations coupled in terms of first derivatives. The problem of non-self-adjointness of the three-body Hamiltonian is solved by cutting off the effective interaction at small distances. This method of regularization implicitly introduces three-body forces. In this case, the cut-off radius plays the role of the free parameter.

Three-body forces are introduced more explicitly in the effective field theory (EFT) [10], causing an integral equation that is similar to the STM equation, but contains artificial terms. A free parameter enters the phenomenological terms

\footnotetext{
*e-mail: penkov@thsun1.jinr.ru penkov@inp.kz
}

†e-mail: sandhas@physik.uni-bonn.de 
of the kernel of the integral equation and the free terms of the integral equation for the scattering problem [10].

Finally, we would like to draw attention to the two-pole $t$ matrix model for the description of three-boson systems [11] which employs the Alt-Grassberger-Sandhas (AGS) [12] version of the Faddeev equations [13]. The position of the second pole on the unphysical sheet is treated in this model as a parameter of the integral equation. When the position of the second pole tends to infinity, the STM equations are reproduced [11]. This method deals with compact equations and is well suited for numerical calculations, but is of little use for analytic considerations.

\section{FORMALISM}

In the present paper we follow another strategy. Transforming the STM equations into differential equations allows us to take into account the Danilov condition quite directly. For this purpose, we construct an infinite system of differential equations in momentum space with a very simple relation between the equations.

The homogeneous part of the STM equation for the elastic scattering amplitude [5] of a boson of mass $m$ on a two-boson bound state of energy $\varepsilon=-\varkappa^{2} / m$ can be represented in a form convenient for further analysis,

$$
F\left(k_{i}\right)=\frac{2}{\pi} \int_{0}^{\infty} \ln \frac{k_{i}^{2}+k^{2}+k_{i} k+\lambda^{2}}{k_{i}^{2}+k^{2}-k_{i} k+\lambda^{2}} \frac{F(k) d k}{\sqrt{\lambda^{2}+k^{2} 3 / 4}-\varkappa},
$$

where $\lambda^{2}=-m Z$. The function $F(k)$ is related to the wave function of the three-boson system $\varphi(k)$ via $F(k)=$ $k\left(\sqrt{-m Z+k^{2} 3 / 4}-\varkappa\right) \varphi(k)$. It should be noted that Eq. (4) implies $F(-k)=-F(k)$.

For further transformations of (4) it is convenient, following [6], to substitute the variables $k=\frac{\lambda\left(t^{2}-1\right)}{\sqrt{3} t}(F(k(t)) \equiv F(t))$ and use the Mellin transformation $\mathcal{F}(s)=\int_{0}^{\infty} t^{s-1} F(t) d t$

$$
\mathcal{F}(s)=L(s) \mathcal{F}(s)+L(s) 2 \frac{\varkappa}{\lambda} \int_{0}^{\infty} d t \frac{t^{s-1}}{\frac{t^{2}+1}{t}-2 \frac{\varkappa}{\lambda}} F(t),
$$

where

$$
L(s)=\frac{8}{\sqrt{3}} \frac{\sin \left(\frac{\pi}{6} s\right)}{s \cos \left(\frac{\pi}{2} s\right)} .
$$

By inverting $(1-L(s))$, Eq. (5) goes over into

$$
\mathcal{F}(s)=\mathcal{F}_{\text {as }}(s)+\frac{L(s)}{1-L(s)} 2 \frac{\varkappa}{\lambda} \int_{0}^{\infty} d t \frac{t^{s-1}}{\frac{t^{2}+1}{t}-2 \frac{\varkappa}{\lambda}} F(t) .
$$

Here, the general solution of

$$
(1-L(s)) \mathcal{F}_{\text {as }}(s)=0
$$

for $s= \pm i \mu_{0}\left(\mu_{0}=1.00623 \ldots\right)$ had to be added. We recall that Eq.(7) was used by Minlos and Faddeev [6] to study the STM equation spectra. In the variable $t$ the solution is

$$
F_{a s}(t) \propto \sin \left(\mu_{0} \ln t\right) .
$$

When carrying out the backward Mellin transformation we see that the function $F$ can be written as a sum

$$
F=F_{0}+\sum_{i=1}^{\infty} F_{i}
$$

over the residues of the function $(1-L(s))^{-1}$. The term $F_{0}$ is generated by the poles at the points $s= \pm i \mu_{0}$ and the terms $F_{i}$ correspond to the real positive solutions of the equation $1-$ $L\left(X_{i}\right)=0$. It can be shown that the components of the function $F$ satisfy the differential equations

$$
\begin{aligned}
& t\left(\frac{d}{d t} t \frac{d}{d t} F_{0}\right)+\mu_{0}^{2} F_{0}+\frac{2 \mu_{0}}{D\left(\mu_{0}\right)} \frac{2 \frac{\varkappa}{\lambda}}{\frac{t^{2}+1}{t}-2 \frac{\varkappa}{\lambda}} F(t)=0, \\
& t\left(\frac{d}{d t} t \frac{d}{d t} F_{i}\right)-X_{i}^{2} F_{i}+\frac{2 X_{i}}{D\left(X_{i}\right)} \frac{2 \frac{\varkappa}{\lambda}}{\frac{t^{2}+1}{t}-2 \frac{\varkappa}{\lambda}} F(t)=0,
\end{aligned}
$$

where $D\left(X_{i}\right)=\left.(1-L(s))^{\prime}\right|_{s=X_{i}}$ and $i D\left(\mu_{0}\right)=\left.(1-L(s))^{\prime}\right|_{s=i \mu_{0}}$. Boundary conditions at $t=1,(k=0)$ follow from the abovementioned antisymmetry of $F(k)$, i.e., $\left.F_{i}(t)\right|_{t=1}=0 ; i=$ $0,1, \ldots$. At large values of the argument one can use either the property of boundedness, or the asymptotic form following from condition (2): $\left.F(t)\right|_{t \rightarrow \infty} \rightarrow \sin \left(\mu_{0} \ln t+\delta\right)$. Comparing the Danilov condition (3) with this asymptotic form of $F$ we get an extra condition for the spectrum

$$
\delta+\mu_{0} \ln (\sqrt{3} / \lambda)=\operatorname{const}(Z)+\pi n
$$

It can be verified that the asymptotics of $F$ coincides with that of $F_{0}$ and the contribution of the remaining terms decreases at infinity. At $\varkappa / \lambda=0$ the system of equations 910 , becomes uncoupled and has a simple solution, $F=F_{0}=$ $\sin \left(\mu_{0} \ln (t)\right)$, which gives, together with condition 111, the spectrum $E_{n}=E_{0} \exp (-2 \pi n)$. This spectrum contains the tree-body collapse at large $\lambda$ for $n<0$ [6], and Efimov's concentration towards the point $Z=0$ at extremely small $\varkappa$ for $n>0$. At small finite $\varkappa$ we can restrict ourselves to Eq. (9) that belongs to a well known class of differential equations (Heun) [14].

\section{RESULTS}

Let us first demonstrate the convergence of the solutions for an increasing number $N$ of equations 910 . To this end, we denote the solution of this system of equations by $F_{R}$ which, upon substitution into the right-hand side of (4), gives the function $F_{L}$. The degree of proximity of $F_{R}$ and $F_{L}$ shows to what extent the solution of the system 9110 is close to that of the integral equation. Figure 1 shows this convergence for $N=1,2,3$. The energy parameter $Z / \varepsilon=(\lambda / \varkappa)^{2}$ was chosen to be 1.57. This value corresponds to the calculations [15] for the binding energy of a helium trimer. In this way we fix the free parameter characteristically occurring in all STM treatments. The good convergence achieved already for $N=3$ indicates the efficiency of our differential equations approach.

Instead of making a similar consideration for the boundstate spectrum, we compare our present results with alternative calculations. In this context we use the binding energies 


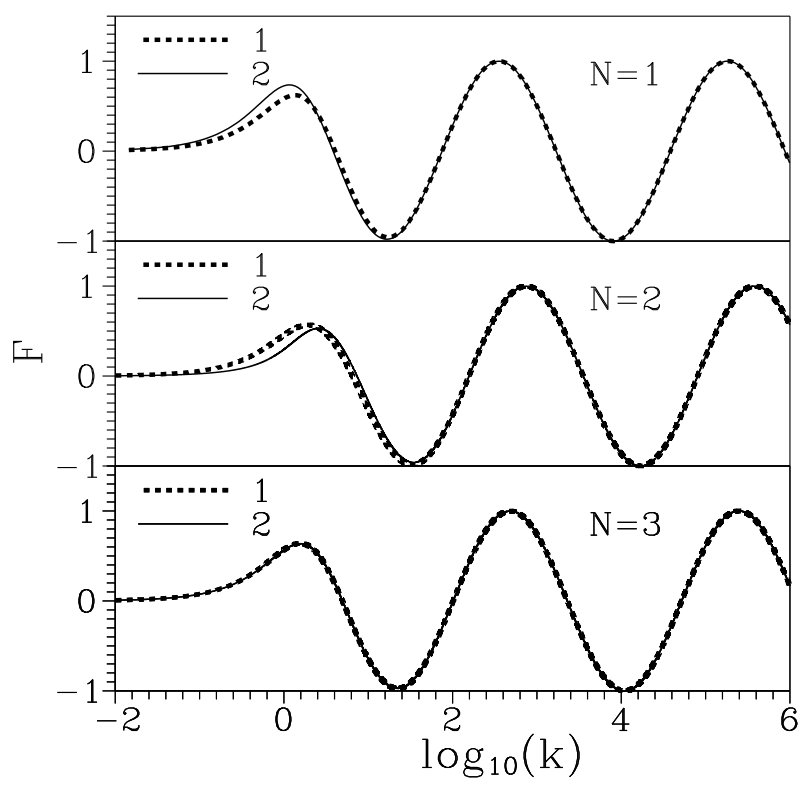

FIG. 1: Convergence of the solutions of Eqs. 910 for increasing $N$ : Curve 1 shows $F_{L}$, curve 2 shows $F_{R}$

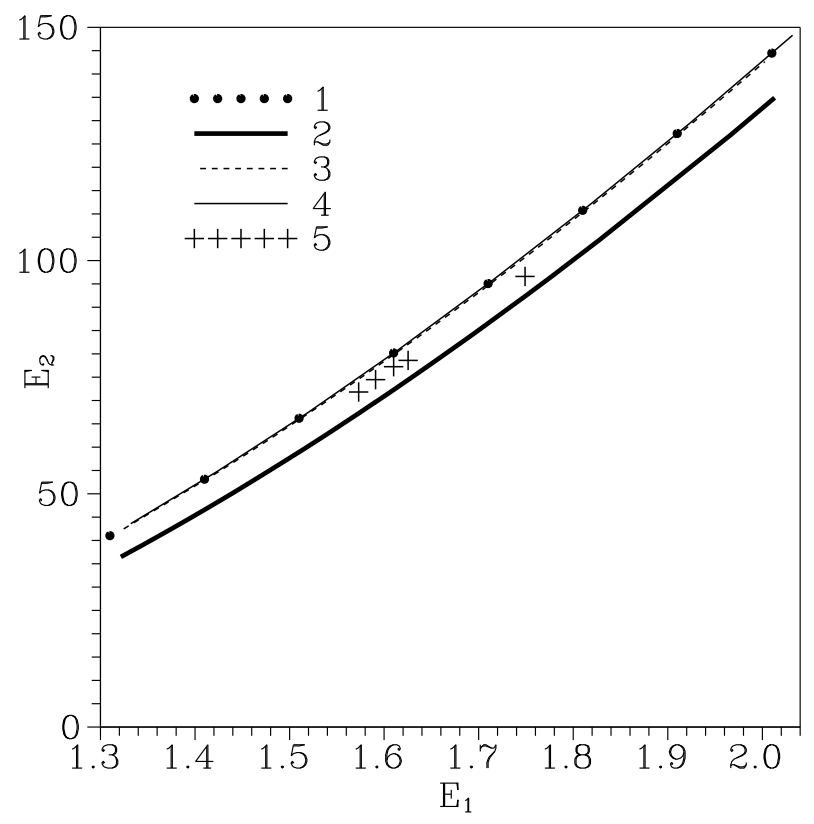

FIG. 2: Trimer binding energies: 1 calculations by Eqs. 910; 2 calculations [11]; 3, 4 calculations for three- and four-level trimers; $\mathbf{5}$ calculations [15, 16] for a helium trimer [15, 16] of a helium trimer, obtained in $[15,16]$ for realistic pair potentials, and the calculations of the binding energies via Faddeevtype AGS integral equations in the framework of the abovementioned two-pole pair $t$-matrix [11]. Since, depending on the position of the $t$-matrix pole on the unphysical sheet, one can obtain as many bound trimer states as one wishes, we will label the highest bound state by "1" and the following one by "2".

Figure 2 shows energy $E_{2}$ as a function of energy $E_{1}$ calculated with Eqs. (910) for $N=21$. Also shown are the calculations for a two-level trimer within the two-pole pair $t$ matrix [11], and our calculations in the same model when the parameters of the pair $t$-matrix admit the existence of three and four bound states of a boson trimer. All the energies are given in units of the dimer energy. We see that there is only a rather small difference between the corresponding curves. Quite interesting is the fact that the calculations for a helium trimer [15, 16] with realistic pair potentials lie just between these curves.

Thus, we have demonstrated that Eqs. 910 represent a very efficient tool for calculating three-boson trimers. These equations can also be extended to scattering problems.

The authors are grateful to A.K. Motovilov and E.A. Kolganova for fruitful discussions. This work was supported by the Deutsche Forschungsgemeinschaft (DFG) and the Russian Foundation for Basic Research.
[1] F. Luo et al., J. Chem. Phys. 98, 3564 (1993); W. Schöllkopf and J.P. Toennies, Science 266, 1345 (1994).

[2] P. O. Fedichev, M. W. Reynolds, and G. V. Shlyapnikov, Phys. Rev. Lett. 77, 2921 (1996).

[3] R. A. Minlos and L. D. Faddeev, Dokl. Akad. Nauk. SSSR 141, 1335 (1961) [Sov. Phys. Doklady 6, 1072 (1962)].

[4] G. S. Danilov, Zh. Eksp. Teor. Fiz. 40, 498 (1961) [Sov. Phys.
JETP 13, 349 (1961)].

[5] G. V. Skornyakov and K. A. Ter-Martirosyan, Zh. Eksp. Teor. Fiz. 31, 775 (1956) [Sov. Phys. JETP 4, 648 (1956)].

[6] R. A. Minlos and L. D. Faddeev, Zh. Eksp. Teor. Fiz. 41, 1850 (1961) [Sov. Phys. JETP 14, 1315 (1961)].

[7] L. H. Thomas, Phys. Rev. 47, 903 (1935).

[8] D. V. Fedorov, A. S. Jensen, and K. Riisager, Phys. Rev. C 50, 
2372 (1994).

[9] V. Efimov, Yad. Fiz. 12, 1080 (1970) [Sov. J. Nucl. Phys. 12, 589 (1970)].

[10] P. F. Bedaque, H.-W. Hammer, and U. van Kolck, Phys. Rev. Lett. 82, 463 (1999).

[11] F. M. Pen'kov, JETP 97, 485 (2003).

[12] E. O. Alt, P. Grassberger, and W. Sandhas, Nucl. Phys. B2, 167 (1967); P. Grassberger and W. Sandhas, Nucl. Phys. B2, 181 (1967).

[13] L. D. Faddeev and S. P. Merkuriev, Quantum scattering the- ory for several particle systems (Kluwer Academic Publishers, Dordrecht, 1993).

[14] S. Yu. Slavyanov, W. Lay, Special Function: A Unified Theory Based on Singularities (Oxford University Press, New York, 2000).

[15] V. Roudnev, S. Sofianos, and S. Yakovlev, Lanl e-print physics/0204025 submitted to Few-Body Systems

[16] A. K. Motovilov, W. Sandhas, S. A. Sofianos, and E. A. Kolganova, Eur. Phys. J. D 13, 33 (2001). 